\title{
Composite MRI measures and short-term disability in patients with clinically isolated syndrome suggestive of MS
}

Giulia Bommarito, Alessandro Bellini, Matteo Pardini, Claudio Solaro, Luca Roccatagliata,
Alice Laroni, Elisabetta Capello, Giovanni Luigi Mancardi, Antonio Uccelli and Matilde Inglese

\section{Abstract}

Background: The use of composite magnetic resonance imaging (MRI) measures has been suggested to better explain disability in patients with multiple sclerosis (MS). However, little is known about the utility of composite scores at the earliest stages of the disease.

Objective: To investigate whether, in patients with clinically isolated syndrome (CIS), a composite MRI measure, rather than the single metrics, would explain conversion to MS and would better correlate with disability at baseline and at 1 year of follow-up.

Methods: Corticospinal tract (CST), corpus callosum (CC) and optic radiation (OR) volume, fractional anisotropy (FA), and mean diffusivity (MD) values were measured in 27 CIS patients and 24 healthy controls (HCs). Z-scores of FA, MD, and tract volume measures were calculated in patients, based on the corresponding measures obtained from $\mathrm{HCs}$, and then combined in a composite score for each tract. Correlations between Z-scores at baseline and both the Expanded Disability Status Scale (EDSS) at baseline and at follow-up (FU-EDSS) were investigated.

Results: Only CST, CC, and OR composite scores as well as the CST volume were significantly associated with FU-EDSS ( $p=0.005, p=0.007, p=0.020$, and $p=0.010$, respectively).

Conclusion: The combination of MRI measures rather than the individual metrics better captured the association between tissue damage in both the CC, OR and CST and short-term follow-up disability.

Keywords: Multiple sclerosis, clinically isolated syndrome, MRI, disability

Date received: 6 December 2016; revised: 17 March 2017; accepted: 19 March 2017

\section{Introduction}

The prediction of short-term disability progression and the response to treatments are still challenging for patients with multiple sclerosis (MS). Both clinical and radiological measures of outcome present limitations, including the inadequacy of the most widely used scale for clinical assessment (i.e. the Expanded Disability Status Scale; EDSS) and the incomplete information about global damage gathered from single or conventional magnetic resonance imaging (MRI) parameters. Although a relationship between MRI conventional measures and clinical relapses has been confirmed, conventional MRI metrics are weakly associated with disability or disease progression. ${ }^{1}$

In approximately $85 \%$ of cases, MS presents with a relapse that is an isolated neurological event, defined as clinically isolated syndrome (CIS). ${ }^{2}$ About $68 \%$ of CIS patients convert to Clinically Definite Multiple Sclerosis (CDMS) or MRI-only MS, according to 2010 McDonald criteria within 6 years of onset. ${ }^{3}$ Although MRI T2 lesion load and changes during the first 5 years have a role in predicting long-term disease progression, ${ }^{4}$ these metrics do not provide a comprehensive and reliable prognostic measure for short-term disability accrual in CIS patients. So far, only a few studies have investigated the role of advanced MRI techniques in the early stages of the disease. 5,6

It has been suggested that the use of composite MRI measures could better describe the relationship between damage in selected brain regions and neurological impairment. ${ }^{7}$ In particular, the use of the diffusion tensor imaging (DTI), which provides
Multiple Sclerosis Journal 2018, Vol. 24(5) 623-631 DOI: $10.1177 /$ 1352458517704077

(C) The Author(s), 2017. Reprints and permissions: http://www.sagepub.co.uk/ journalsPermissions.nav
Correspondence to: $M$ Inglese

Departments of Neurology, Radiology and Neuroscience, Icahn School of Medicine at Mount Sinai, Annenberg 14, Box 1137, One Gustave L. Levy Place, New York, NY 10029, USA. matilde.inglese@mssm.edu Giulia Bommarito Matteo Pardini

Alice Laroni

Elisabetta Capello Giovanni Luigi Mancardi Antonio Uccelli Department of Neurology, Rehabilitation, Ophthalmology, Genetics, Maternal and Child Health (DINOGMI), University of Genoa, Genoa, Italy/IRCCS AOU San Martino-IST, Genoa, Italy Alessandro Bellini Department of Neurology, Rehabilitation, Ophthalmology, Genetics, Maternal and Child Health (DINOGMI), University of Genoa, Genoa, Italy/IRCCS AOU San Martino-IST, Genoa, Italy/UOC Fisica Medica e Sanitaria, IRCCS AOU San Martino-IST, Genoa, Italy

Claudio Solaro

Neurology Unit, Department of Head and Neck, PA Micone Hospital, ASL3 Genovese, Genoa, Italy

Luca Roccatagliata Department of Health Science (DISSAL), University of Genoa, Genoa, Italy/IRCCS AOU San Martino-IST, Genoa, Italy Matilde Inglese Department of Neurology, Rehabilitation, Ophthalmology, Genetics, Maternal and Child Health (DINOGMI), University of Genoa, Genoa, Italy/IRCCS AOU San Martino-IST, Genoa, Italy/Departments of Neurology, Radiology and Neuroscience, Icahn School of Medicine at Mount Sinai, New York, NY, USA 
information on brain tissue microstructural integrity, could be complementary to volumetric measures in the assessment of the major white matter (WM) tracts damage. Histopathological findings indicate that acute axonal damage occurs since the early stages of the disease ${ }^{8}$ and DTI studies have revealed that WM tracts, such as the corpus callosum (CC) and the corticospinal tract (CST), are frequently affected by the disease pathology in CIS patients. ${ }^{9}$ Indeed, as shown by Lin et al., both volumetric and diffusion tensor metrics of CC differed between CIS patients and controls ${ }^{10}$ and volume loss of the CC predicted conversion to MS. ${ }^{5,11}$ Conventional MRI studies have also shown that, in patients with greater disability, T2 lesions were more likely localized in optic radiation (OR) and left internal capsule. ${ }^{12}$ However, in these studies, there was no attempt to combine single MRI metrics in a composite score and test its clinical impact. The aim of our study was to investigate whether composite MRI measures of specific major WM tracts, such as the CST, the OR, and the CC, compared with individual MRI-derived metrics, would explain conversion to MS and would improve the correlation with clinical disability over short-term follow-up in patients with CIS.

\section{Materials and methods}

\section{Participants}

The study was approved by the Local Ethics Committee and all participants gave written informed consensus prior to enrollment.

Patients presenting with a first neurological event, suggestive of a demyelinating disease, were consecutively recruited. The diagnosis was made upon clinical examination and supported by radiological and cerebrospinal fluid (CSF) findings, according to McDonald's criteria 2010. Patients were excluded if they had a history of any previous neurological sign or symptom that could possibly be interpreted as a demyelinating event or if they presented a second relapse before the baseline visit, pregnancy, an active major organ disease, or a serious mental disorder. Patients entered the study within 75 days from their first clinical event and MRI examination at baseline was performed at least 1 month after steroid administration. All patients underwent clinical assessment, including EDSS, every 6 months, up to 12 month.

Healthy volunteers without any neurological disease or other comorbidities served as healthy controls (HCs).

\section{MRI acquisition}

All subjects underwent MRI at baseline on a $1.5 \mathrm{~T}$ scanner (SignaHDxt scanner, GE Medical Systems), using an 8-channel transmit/receive head coil and including the following sequences: (1) spin-echo dualecho proton density-weighted and T2-weighted (TR/ TE1/TE2: 2120/38.2/102 ms, 72 contiguous 4-mmthick axial slices); (2) three-dimensional sagittal Fast Spoiled Gradient Recalled echo (FSPGR)T1-weighted sequence (TR/TE: 9.6/4.1 ms; Inversion Time 500; 152 contiguous sagittal slices; voxel size: $1.2 \mathrm{~mm}$, isotropic; field of view 240; matrix size $256 \times 256$ ); (3) DTI was obtained using a single-shot spin-echo echo-planar imaging (TR/TE: 14,000/93.4 ms, slice thickness 2.5 , gradients applied to 15 non-collinear directions; $b$-value $=1000 \mathrm{~s} / \mathrm{mm}^{2} ; 5$ volumes with $b$-values $=0$ ); and (4) spin-echo T1-weighted after injection of a single dose of Gadoteridol $(0.2 \mathrm{~mL} / \mathrm{kg})$ (TR/TE 520/20 ms, 36 contiguous 4-mm-thick axial slices).

\section{MRI analysis: lesion identification and DTI} preprocessing

WM T2, T1, and gadolinium T1 (GD T1) lesion volumes (LV) were measured on the T2/Proton Density (PD)-weighted and pre- and post-contrast T1-weighted images, using a semi-automated technique based on user-supervised local thresholding (Jim version 6; Xinapse Systems, http://www.xinapse.com).

Volumes of the whole brain (WBV), gray matter (GMV), white matter (WMV), and CSF, normalized for subject head size, were estimated with SIENAX, ${ }^{13}$ part of FSL, on the lesion-filled three-dimensional (3D) T1-weighted images, as described in Battaglini et al., ${ }^{14}$ for both patients and healthy subjects. Brain parenchymal fraction (BPF) was calculated by adding WMV and GMV and dividing the resulting value for the sum of WMV, GMV, and the volume of CSF.

Maps of fractional anisotropy (FA) and mean diffusivity (MD) were obtained for all subjects by fitting of the diffusion tensor images after eddy current correction and brain extraction, using DTIFit (FMRIB Diffusion Toolbox, part of the FMRIB Software Library, FSL; http://www.fmrib.ox.ac.uk/fsl).

Volume, FA, and MD values of the CC of the left and right CST and of the left and right OR were computed for each subject with the following procedure: (1) T1 images were linearly registered to the Montreal Neurological Institute (MNI) 152 T1 $2 \mathrm{~mm}$ template brain using FMRIB's Linear Imager Registration Tool (FLIRT) ${ }^{15}$ version 6.0 with affine 


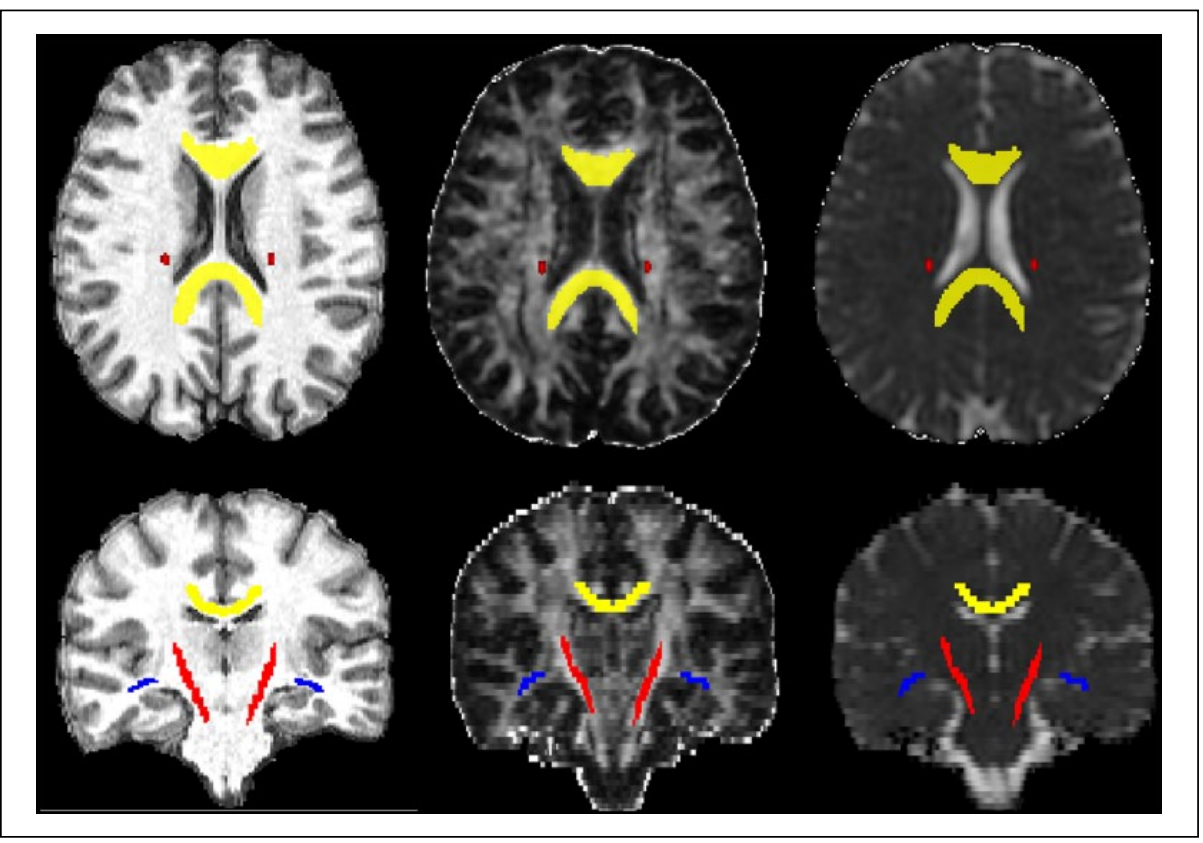

Figure 1. Selected axial (top row) and coronal (bottom row) sections of 3D FSPGR T1-weighted (left column), FA map (middle column), and MD map (right column) images from a CIS patients, with superimposed co-registered JHU atlas masks of CC (yellow), right and left CST (red), and right and left OR (blue).

transformation and correlation ratio cost function; (2) the matrix from the previous registration was used to perform a non-linear registration from $\mathrm{T} 1$ to MNI 152 space using FMRIB's Non-linear Imager Registration Tool (FNIRT); ${ }^{16}$ (3) non-diffusion weighted $\mathrm{b} 0$ average images were linearly registered to the T1 dataset, using FLIRT, with affine transformation and mutual information cost function; (4) the warp of the non-linear registration from T1 to MNI 152 and the matrix of linear registration from b0 to T1 were both inverted; (5) left and right CST, left and right $\mathrm{OR}$, and $\mathrm{CC}$ masks were obtained from the JHU white-matter tractography atlas, the Juelich histological atlas, ${ }^{17}$ and the JHU ICBM-DTI-81 whitematter labels atlas; ${ }^{18}$ (6) CST, OR, and CC masks were translated from atlas space to $\mathrm{T} 1$ space using the inverted warp and to b0 space applying the inverted matrix; (7) all masks were visually inspected for correct positioning. CC, OR, and CST masks translated to native $\mathrm{T} 1$ space were used to derive mean volume values of each tract; $\mathrm{CC}, \mathrm{OR}$, and CST masks translated to b0 space were applied to FA and MD maps to measure the respective mean FA and MD values for each tracts (Figure 1). Masks of the white matter lesion region of interest (ROI) identified on the T2-weighted images were used to evaluate the presence of focal lesions within the $\mathrm{CC}$, the OR, or the CST. For each patient, T2-weighted images were co-registered to the native T1-weighted space using FLIRT with traditional transformation and mutual information cost function; the matrix was then applied to the lesion mask using FLIRT with nearest-neighbor interpolation. Lesion, OR, CST, and CC masks were visualized in T1 space to compute the lesion number within each tract. Finally, after segmenting the lesions out, the corresponding $\mathrm{CC}, \mathrm{OR}$, and CST masks without lesions were translated to native $\mathrm{T} 1$ space, to derive mean volumes of each normal-appearing white matter (NAWM) tract, and to the FA and MD maps, to derive the respective mean FA and MD values for each NAWM tract.

\section{Z-scores calculation}

$\mathrm{Z}$-scores were calculated for $\mathrm{FA}\left(\mathrm{Z}_{\mathrm{FA}}\right), \mathrm{MD}\left(\mathrm{Z}_{\mathrm{MD}}\right)$, and volume $\left(Z_{\mathrm{VOL}}\right)$ measures for all CIS subjects, based on controls mean values (meanHC) and standard deviation data (SDHC), as follows

$$
\begin{aligned}
& \mathrm{Z}_{\mathrm{FA}}=\frac{\left(\mathrm{FA}_{\mathrm{CIS}}-\mathrm{FA}_{\text {meanHC }}\right)}{\mathrm{FA}_{\mathrm{SDHC}}} \\
& \mathrm{Z}_{\mathrm{VOL}}=\frac{\left(\mathrm{VOL}_{\mathrm{CIS}}-\mathrm{VOL}_{\text {meanHC }}\right)}{\mathrm{VOL}_{\mathrm{SDHC}}} \\
& \mathrm{Z}_{\mathrm{MD}}=\frac{\left(\mathrm{MD}_{\mathrm{CIS}}-\mathrm{MD}_{\text {meanHC }}\right)}{\mathrm{MD}_{\mathrm{SDHC}}}
\end{aligned}
$$

for $\mathrm{CC}, \mathrm{OR}$, and $\mathrm{CST}$ with and without lesions $\left(Z_{\text {FA-NAWM }}, Z_{\text {VOL-NAWM }}\right.$, and $\left.Z_{\text {MD-NAWM }}\right)$. 
Table 1. Demographic, clinical and conventional, and tract-specific MRI features in patients with clinically isolated syndrome and healthy subjects.

\begin{tabular}{|c|c|c|c|}
\hline & \multicolumn{2}{|c|}{ Patients } & Controls \\
\hline Age & \multicolumn{2}{|c|}{$39.0 \pm 10.9$} & $37.71 \pm 19.5$ \\
\hline Female, $n(\%)$ & \multicolumn{2}{|c|}{$17(63)$} & $11(46)$ \\
\hline Median EDSS (range) at baseline & \multicolumn{2}{|c|}{$1.5(0-3.5)$} & \\
\hline Median EDSS (range) at 1-year follow-up & \multicolumn{2}{|c|}{$1.0(0-3.0)$} & \\
\hline Conversion to MS according to McDonalds $2010^{\mathrm{a}}$ & \multicolumn{2}{|c|}{$19 / 27$} & \\
\hline Number of relapses ${ }^{a}$ & \multicolumn{2}{|c|}{$7 / 27$} & \\
\hline $\mathrm{CSF} \mathrm{OB}+/-$ & \multicolumn{2}{|c|}{$17 ; 8$} & \\
\hline T2-LL (mL) & \multicolumn{2}{|c|}{$1.37 \pm 1.99$} & \\
\hline T1-LL (mL) & \multicolumn{2}{|c|}{$0.14 \pm 0.32$} & \\
\hline $\mathrm{CC}$ & Whole tract & NAWM & WM \\
\hline $\operatorname{MD}\left(10^{-3} \mathrm{~mm}^{2} \mathrm{~s}^{-1}\right)$ & $0.880 \pm 0.054 \dagger$ & $0.880 \pm 0.054 \dagger$ & $0.833 \pm 0.080$ \\
\hline FA & $0.589 \pm 0.054$ & $0.590 \pm 0.053$ & $0.621 \pm 0.070$ \\
\hline Vol (mL) & $18.026 \pm 3.228$ & $17.950 \pm 3.227$ & $17.726 \pm 2.051$ \\
\hline $\mathrm{CST}$ & Whole tract & NAWM & WM \\
\hline $\operatorname{MD}\left(10^{-3} \mathrm{~mm}^{2} \mathrm{~s}^{-1}\right)$ & $0.749 \pm 0.032 \ddagger$ & $0.749 \pm 0.031 \ddagger$ & $0.695 \pm 0.054$ \\
\hline FA & $0.632 \pm 0.040$ & $0.633 \pm 0.040$ & $0.668 \pm 0.074$ \\
\hline Vol (mL) & $4.881 \pm 0.780$ & $4.853 \pm 0.823$ & $4.847 \pm 0.631$ \\
\hline OR & Whole tract & NAWM & WM \\
\hline $\operatorname{MD}\left(10^{-3} \mathrm{~mm}^{2} \mathrm{~s}^{-1}\right)$ & $0.822 \pm 0.052 \ddagger$ & $0.821 \pm 0.049 \ddagger$ & $0.756 \pm 0.055$ \\
\hline FA & $0.432 \pm 0.052$ & $0.432 \pm 0.051$ & $0.483 \pm 0.092$ \\
\hline Vol (mL) & $2.244 \pm 0.372$ & $2.234 \pm 0.375$ & $2.357 \pm 0.308$ \\
\hline \multicolumn{4}{|c|}{$\begin{array}{l}\text { EDSS: Expanded Disability Status Scale; MS: multiple sclerosis; CSF: cerebrospinal fluid; T2-LL: lesion load on T2-weighted } \\
\text { sequence; T1-LL: lesion load on T1-weighted sequence; CC: corpus callosum; CST: corticospinal tract; OR: optic radiation; MD: } \\
\text { mean diffusivity; FA: fractional anisotropy; NAWM: normal-appearing white matter; WM: white matter. } \\
\text { Values are reported as mean and standard deviation, unless otherwise specified. } \\
\text { aWithin 1 year of follow-up. } \\
{ }^{t} p<0.05 \text {. } \\
{ }^{t} p<0.01 \text {. }\end{array}$} \\
\hline
\end{tabular}

Volume, FA, and MD Z-scores values were subsequently combined to obtain a composite Z-score

$$
\begin{aligned}
& Z_{\text {COMP }}=Z_{\mathrm{FA}}-Z_{\mathrm{MD}}+Z_{\mathrm{VOL}}, \\
& Z_{\text {COMP-NAWM }}=Z_{\text {FA-NAWM }}-Z_{\text {MD-NAWM }}+Z_{\text {VOL-NAWM }}
\end{aligned}
$$

\section{Statistical analysis}

Mann-Whitney test was used to assess differences in MRI parameters between patients who converted to MS within 1-year follow-up (FU) and patients who did not convert and to assess differences in MRI parameters between patients and HCs. Spearman rank correlation test was used to assess the correlation between single and composite MRI scores and baseline and 1-year follow-up EDSS scores. Nonparametric partial correlation was used to correct for $\mathrm{BPF}$ and treatment variables. Statistical analysis was performed with SPSS (IBM SPSS Statistics 21) and statistical significance reported at $p<0.05$.

Correction for multiple comparisons was achieved with the false discovery rate (FDR) method. ${ }^{19}$

\section{Results}

\section{Demographics and clinical data}

Demographic and clinical data at 1 year of follow-up were available for 27 patients (17 females, 10 males; mean age, $39 \pm 10.9$ years) and are reported in Table 1 . A total of 24 healthy volunteers (11 females, 13 males; mean age, $37.71 \pm 19.5$ years) served as controls. In all, 12 out of 27 CIS patients presented with optic neuritis, 7 with brainstem and 6 with spinal cord syndrome.

A total of 19 patients converted to MS according to McDonald criteria 2010 and 7 out of 27 patients presented a clinical relapse within 1 year of follow-up.

In all, 12 out of 27 patients started a treatment within 1 year of observation, at a variable lapse of time from the baseline assessment. In particular, 10/27 patients started a first-line disease-modifying therapy (interferon beta or dimethyl fumarate), 2 of them were subsequently switched to a second-line agent (natalizumab or fingolimod); 2 patients presented an aggressive 
course of the disease, therefore a second-line drug was used as initial treatment.

Median EDSS was 1.5 (range, 0-3.5) at baseline and 1.0 (range, $0-3$ ) at 1-year follow-up. EDSS worsening (defined as an increase of 1 point compared with the initial EDSS score or an increase of 1.5 points if the initial score was 0 ) was detected in four patients (confirmed at the 6-month and 1-year follow-up visit).

\section{Global and regional lesion load}

Mean T2-LV and T1-LV are reported in Table 1. T2-LV was significantly higher in patients who converted to MS within 1 year $(p<0.01)$.

T2-LV and T1-LV significantly correlated with EDSS at 1 -year FU ( $r=0.447, p=0.02$ and $r=0.659, p<0.01$, respectively) but not with EDSS at baseline.

No significant association was found between WBV, GMV, and WMV and EDSS at baseline or at 1-year FU.

In all, 9 out of 27 patients presented T2-W lesions in the $\mathrm{CC}$ (mean number, 2.3; range, 1-5), 5 patients presented T2-W lesions in the left or right OR (mean number, 2.8; range, 1-5), and 5 patients presented $\mathrm{T} 2-\mathrm{W}$ lesions in the left or the right CST (mean number, 1.0; no more than 1 lesion detected in either left or right CST per each patient).

\section{CC and CST MRI metrics}

Mean and standard deviation of MRI parameters for both patients and HCs are reported in Table 1.

MD values of CC, CST, and OR were significantly different between patients and HCs groups, for both the entire tract and the tract without lesions $(p=0.032$ for both CC and NAWM CC; $p<0.01$ for both CST and NAWM CST; $p<0.01$ for both OR and NAWM OR). A trend of significance was also found for FA values of the three tracts ( $p$ values ranging from 0.061 to 0.081 ).

None of the CC, OR, or CST Z-scores differed between patients who had converted or not to MS.

None of the single or composite MRI metrics from both $\mathrm{CC}$, OR, and $\mathrm{CST}$ with and without lesions (NAWM CC and CST) was significantly associated with baseline EDSS scores ( $p$ values ranging from 0.1 to 0.9 ).

Correlations between CC, OR, and CST MRI metrics and EDSS scores at 1-year FU are reported in Table 2.
Among the CC MRI measures, a statistically significant correlation was found between the composite score and EDSS score at 1-year FU, for both CC with and without lesions (NAWM CC) $(r=-0.528$, $p=0.005$ and $r=-0.526, p=0.005$ for $Z_{\mathrm{COMP}}$ and $\mathrm{Z}_{\text {COMP-NAWM}}$, respectively). No significant correlations were found with isolated volume, FA, and MD $\mathrm{CC}$ measures.

Among the CST MRI measures, a statistically significant correlation was found between the composite score for both CST with and without lesions (NAWM CST) $(r=-0.506, p=0.007$ and $r=-0.492, p=0.009$ for $Z_{\text {COMP }}$ and $Z_{\text {COMP-NAWM }}$, respectively) and between the single volume score for both CST with and without lesions (NAWM CST) $(r=-0.461, p=0.016$ and $r=-0.495, p=0.009$ for $\mathrm{Z}_{\mathrm{VOL}}$ and $\mathrm{Z}_{\mathrm{VOL}-\mathrm{NAWM}}$, respectively) and EDSS at 1-year FU.

Among the OR MRI measures, a statistically significant correlation was found between the composite score for both OR with and without lesions (NAWM OR) $(r=-0.444, p=0.020$ and $r=-0.433, p=0.024$ for $\mathrm{Z}_{\mathrm{COMP}}$ and $\mathrm{Z}_{\text {COMP-NAWM }}$, respectively) and between the single volume score of NAWM OR $(r=-0.418$, $p=0.030$ for $\left.\mathrm{Z}_{\mathrm{VOL}-\mathrm{NAWM}}\right)$ and EDSS at 1 -year FU.

Correlations remained significant after correction for $\mathrm{BPF}$ ( $p=0.005$ and $p=0.006$ for $\mathrm{Z}_{\mathrm{COMP}}$ and $\mathrm{Z}_{\text {COMP-NAWM }}$ of $\mathrm{CC}$, respectively; $p=0.010$ for $\mathrm{Z}_{\mathrm{COMP}}, \mathrm{Z}_{\text {COMP-NAWM}}$, and $\mathrm{Z}_{\mathrm{VOL}-\mathrm{NAWM}}$ and $p=0.018$ for $\mathrm{Z}_{\mathrm{VOL}}$ of CST, respectively; $p=0.014, p=0.017$, and $p=0.018$ for $Z_{\text {COMP, }}$ $Z_{\text {COMP-NAWM }}$, and $Z_{\text {VOL-NAWM }}$ of OR, respectively) or after adding disease treatment as a covariate $(p=0.032$ and $p=0.033$ for $Z_{\text {COMP }}$ and $Z_{\text {COMP-NAWM }}$ of $\mathrm{CC}$, respectively; $p=0.031$ for both $Z_{\text {COMP }}$ and $\mathrm{Z}_{\text {COMP-NAWM }}$ of CST; $p=0.032$ for $Z_{\mathrm{VOL}}$ and $p=0.020$ for $Z_{\text {VOL-NAWM }}$ of CST; $p=0.033$ and $p=0.037$ for $\mathrm{Z}_{\text {COMP }}$ and $\mathrm{Z}_{\text {COMP-NAWM }}$ of OR, respectively).

Correlations between EDSS at 1-year FU and both $\mathrm{Z}_{\text {COMP }}$ and $\mathrm{Z}_{\text {COMP-NAWM }}$ of CST and CC survived the FDR correction.

\section{Discussion}

Our study suggests that composite MRI measures of selected white matter tracts, which are preferentially involved in MS, provide a better correlate of shortterm disease progression, even at the early stages of the disease, compared with individual MRI-derived metrics.

In line with previous studies, ${ }^{20}$ we confirmed that a greater $\mathrm{T} 2-\mathrm{LV}$ is associated with conversion to $\mathrm{MS}$ 
Table 2. Correlations between EDSS at 1-year FU and the Z-scores of the corpus callosum, the optic radiation, and the bilateral corticospinal tract with and without lesions (NAWM).

\begin{tabular}{|c|c|c|}
\hline & Corpus callosum & Corpus callosum NAWM \\
\hline $\mathrm{Z}_{\mathrm{MD}}$ & $r=0.162, p=0.420$ & $r=0.162, p=0.420$ \\
\hline $\mathrm{Z}_{\mathrm{FA}}$ & $r=-0.253, p=0.203$ & $r=-0.252, p=0.205$ \\
\hline $\mathrm{Z}_{\mathrm{VOL}}$ & $r=-0.331, p=0.092$ & $r=-0.364, p=0.062$ \\
\hline \multirow[t]{2}{*}{$\mathrm{Z}_{\mathrm{COMP}}$} & $r=-0.528, p=0.005$ & $r=-0.526, p=0.005$ \\
\hline & Bilateral corticospinal tract & Bilateral corticospinal tract NAWM \\
\hline $\mathrm{Z}_{\mathrm{MD}}$ & $r=-0.099, p=0.624$ & $r=-0.099, p=0.624$ \\
\hline $\mathrm{Z}_{\mathrm{FA}}$ & $r=-0.206, p=0.303$ & $r=-0.181, p=0.367$ \\
\hline $\mathrm{Z}_{\mathrm{VOL}}$ & $r=-0.461, p=0.016$ & $r=-0.495, p=0.009$ \\
\hline \multirow[t]{2}{*}{$\mathrm{Z}_{\mathrm{COMP}}$} & $r=-0.506, p=0.007$ & $r=-0.492, p=0.009$ \\
\hline & Optic radiation & Optic radiation NAWM \\
\hline $\mathrm{Z}_{\mathrm{MD}}$ & $r=0.201, p=0.314$ & $r=0.185, p=0.355$ \\
\hline $\mathrm{Z}_{\mathrm{FA}}$ & $r=-0.081, p=0.687$ & $r=-0.078, p=0.698$ \\
\hline $\mathrm{Z}_{\mathrm{VOL}}$ & $r=-0.378, p=0.052$ & $r=-0.418, p=0.030$ \\
\hline $\mathrm{Z}_{\mathrm{COMP}}$ & $r=-0.444, p=0.020$ & $r=-0.433, p=0.024$ \\
\hline
\end{tabular}

within 1-year follow-up (FU). The role of the T2 and T1 lesion load on short-term disability worsening is more controversial and the few available studies have showed only the association of $\mathrm{T} 2$ lesion load with long-term FU disability. ${ }^{21,22}$ Our results support the role of the global burden of damage in determining disability, even on a short-term follow-up; however, considering the lack of support in the literature, even on large sample size previous studies, ${ }^{5}$ the predictive value on a short-term disability for T2-LV and T1-LV should be considered cautiously.

DTI parameters, but not the volume values, differed between patients and healthy subjects; this finding confirms that microstructural damage occurs early in demyelinating disorders.

The most interesting finding of our study is the association between a decrease in the composite $\mathrm{CC}, \mathrm{OR}$, and $\mathrm{CST}_{\mathrm{COMP}}$ scores at baseline and EDSS scores at 1-year FU (CC and CST $Z_{\text {COMP }}$ scores survived after FDR correction). Indeed, only the composite measure showed a correlation between EDSS scores at 1-year $\mathrm{FU}$ in the CC, OR, and CST, while only OR $\mathrm{Z}_{\mathrm{VOL} \text {-NAWM }}$ and CST $Z_{\mathrm{VOL}}$ and $Z_{\mathrm{VOL}-\mathrm{NAWM}}$ - but not CC volumecorrelated with disability at 1 year.

CC damage, in terms of FA changes, has been previously reported in CIS patients ${ }^{10,23,24}$ and the presence of lesions within the $\mathrm{CC}$ as well as the early decrease in CC volume have both been found to be associated with a greater risk of conversion to MS. ${ }^{5,11,25}$ Our study extends previous findings by showing the clinical impact of a composite MRI score of CC damage and its significant correlation with EDSS score at short-term follow-up. Visual pathway is involved in demyelinating disorders: a decrease in OR thickness in patients at the early stages of the disease, when compared to healthy subjects, has been detected; ${ }^{26}$ moreover, altered DTI parameters of the OR, in particular radial diffusivity and FA, correlate with the visual evoked potential latencies. ${ }^{27}$ Like the $\mathrm{CC}$ and the OR, the CST is affected by MS pathology since the earliest stages of the disease. Pagani et al. reported that lesion volume within CST and MD values of the CST NAWM were higher in CIS patients with pyramidal symptoms compared to those without pyramidal dysfunction, even though there was no statistically significant association with measures of clinical disability. ${ }^{28}$ Our results are in line with previous findings in that they confirm the lack of significant correlations between either single or composite MRI scores and EDSS scores at baseline; however, in our study, both CST $Z_{\mathrm{VOL}}$, OR $Z_{\mathrm{VOL}-\mathrm{NAWM}}$ and the composite $\mathrm{CC}$, OR and CST Z-scores were significantly associated with EDSS scores at 1-year follow-up.

Our findings suggest that the integration of MRI measures, reflecting different histopathologic aspects of WM damage, could better explain clinical 
outcome. Indeed, FA and MD provide a quantitative measure of ongoing microstructural damage within the tracts $^{29}$ complementing volumetric measures, which reflect the amount of tissue already lost. Volumetric and microstructural damage of major WM tracts could be the results of the presence of focal MS lesions within the tract itself, extralesional damage characterized by inflammation, gliosis, demyelination, and reduced axonal density of the NAWM within the tracts, ${ }^{30}$ and Wallerian degeneration of axons transected in local and remote lesions. ${ }^{31,32}$ While the damage of the $\mathrm{CC}$, a commissural tract, could depend predominantly on Wallerian degeneration of axons injured in distant lesions, the CST, a projection tract, could be affected predominantly by focal white matter or cortical damage; on the other hand, the visual pathway is damaged both through focal damage and trans-synaptic degeneration. ${ }^{33}$

The correlation with CST and OR, but not CC, volume could reflect the greater efficacy of EDSS in detecting motor or visual disability; otherwise, CC damage could result in a more subtle clinical impairment, such as cognitive deficits, better assessed with other clinical scores.

As expected in this early stage of the disease, the number of WM lesions in the OR, CC, and CST was quite low and only half of the patients presented lesions in either tract, thus, preventing the inclusion of a MRI measure of lesion damage in the composite MRI score. Although the presence of OR, CC, and CST lesions did not seem to be relevant in our group of CIS patients as shown by the similar magnitude and significance of association of $Z_{\text {COMP }}$ and $Z_{\text {COMP-NAWM }}$, we believe that adding the tract lesion component to the composite score could become more relevant in the later stage of the disease.

In our cohort, we did not observe a clinical progression at a group level, in terms of EDSS worsening; as reported in the literature, even in studies with a large cohort of patients, ${ }^{5}$ median EDSS after a short-term follow-up did not increase in CIS patients. The correlation between composite MRI metrics at baseline and EDSS at 1-yeat FU, in spite of the absence of a worsening at a group level, supports the prognostic value of the composite measures even in patients with a minimal disability.

Our study findings have to be interpreted with caution due to the small sample size and the lack of clinical scales of evaluation other than EDSS. Therefore, their confirmation in a larger cohort of patients with a longterm follow-up is warranted. Nonetheless, the use of a composite measure instead of single scores reduces the type I error, with valuable results especially in studies with small sample sizes.

Determining the outcome of patients in terms of disability progression, since disease onset, is still challenging but crucial as more and earlier treatments become available. While conventional MRI parameters are insufficient to address this issue, a composite set of selected and meaningful measures of macroand microscopic tissue damage could improve our understanding of clinical variability.

The combination of different MRI measures, even at the level of a single white matter tract, resulted clinically significant and MRI-derived measures of OR, $\mathrm{CC}$ and CST emerged as simple and reliable early markers of clinical disability.

\section{Declaration of Conflicting Interests}

The author(s) declared the following potential conflicts of interest with respect to the research, authorship, and/or publication of this article: Dr Giulia Bommarito has nothing to disclose. Dr Matteo Pardini has nothing to disclose. Alessandro Bellini has nothing to disclose. Dr Luca Roccatagliata has nothing to disclose. Dr Elisabetta Capello has nothing to disclose. Dr Alice Laroni has nothing to disclose. Dr Claudio Solaro served as a member of the advisory board of the following companies: Biogen Idec and Merck Serono; received speaking honoraria from Bayer Schering, Biogen Idec, Merck Serono, Teva, Almirall, and Sanofi Genzyme and research grants and support by the FISM (Fondazione Italiana Sclerosi Multipla). Dr Giovanni Luigi Mancardi has received honoraria for lecturing, travel expenses for attending meetings, and financial support for research from Bayer Schering, Biogen Idec, Sanofi Aventis, Teva, Genzyme, and Merck Serono Pharmaceuticals. Dr Antonio Uccelli has received consulting honoraria and/or speaker fees and basic science study grants from Biogen Idec; consulting honoraria and/or speaker fees from Genzyme, Roche, Sanofi Aventis, and Teva; consulting honoraria and/or speaker fees and a basic science study grant from Novartis; consulting honoraria and/or speaker fees and a basic science study grant from Merck Serono. Dr Matilde Inglese has received research grants from the NIH, NMSS, Novartis Pharmaceuticals, and Teva Neuroscience.

\section{Funding}

The author(s) received no financial support for the research, authorship, and/or publication of this article. 


\section{References}

1. Cohen JA, Reingold SC, Polman $\mathrm{CH}$, et al. Disability outcome measures in multiple sclerosis clinical trials: Current status and future prospects. Lancet Neurol 2012; 11(5): 467-476

2. Miller DH, Chard DT and Ciccarelli O. Clinically isolated syndromes. Lancet Neurol 2012; 11(2): 157-169.

3. Brownlee WJ, Swanton JK, Altmann DR, et al. Earlier and more frequent diagnosis of multiple sclerosis using the McDonald criteria. J Neurol Neurosurg Psychiatry 2015; 86(5): 584-585.

4. Fisniku LK, Brex PA, Altmann DR, et al. Disability and T2 MRI lesions: A 20-year follow-up of patients with relapse onset of multiple sclerosis. Brain 2008; 131(3): 808-817.

5. Uher T, Horakova D, Kalincik T, et al. Early magnetic resonance imaging predictors of clinical progression after 48 months in clinically isolated syndrome patients treated with intramuscular interferon $\beta-1 \mathrm{a}$. Eur J Neurol 2015; 22(7): 1113-1123.

6. Di Filippo M, Anderson VM, Altmann DR, et al. Brain atrophy and lesion load measures over 1 year relate to clinical status after 6 years in patients with clinically isolated syndromes. J Neurol Neurosurg Psychiatry 2010; 81(2): 204-208.

7. Pardini M, Yaldizli Ö, Sethi V, et al. Motor network efficiency and disability in multiple sclerosis. Neurology 2015; 85(13): 1115-1122.

8. Kuhlmann T, Lingfeld G, Bitsch A, et al. Acute axonal damage in multiple sclerosis is most extensive in early disease stages and decreases over time. Brain 2002; 125(Pt 10): 2202-2212.

9. Raz E, Cercignani M, Sbardella E, et al. Clinically isolated syndrome suggestive of multiple sclerosis: Voxelwise regional investigation of white and gray matter. Radiology 2010; 254(1): 227-234.

10. Lin F, Yu C, Liu Y, et al. Diffusion tensor group tractography of the corpus callosum in clinically isolated syndrome. AJNR Am J Neuroradiol 2011; 32(1): 92-98.

11. Kalincik T, Vaneckova M, Tyblova M, et al. Volumetric MRI markers and predictors of disease activity in early multiple sclerosis: A longitudinal cohort study. PLoS ONE 2012; 7(11): 1-8.

12. Dalton CM, Bodini B, Samson RS, et al. Brain lesion location and clinical status 20 years after a diagnosis of clinically isolated syndrome suggestive of multiple sclerosis. Mult Scler 2012; 18(3): 322-328.

13. Smith SM, Zhang Y, Jenkinson M, et al. Accurate, robust, and automated longitudinal and crosssectional brain change analysis. Neuroimage 2002; 17(1): 479-489.
14. Battaglini M, Jenkinson $\mathrm{M}$ and De Stefano N. Evaluating and reducing the impact of white matter lesions on brain volume measurements. Hum Brain Mapp 2012; 33(9): 2062-2071.

15. Jenkinson M, Bannister P, Brady M, et al. Improved optimization for the robust and accurate linear registration and motion correction of brain images. Neuroimage 2002; 17(2): 825-841.

16. Andersson JLR, Jenkinson M and Smith S. Nonlinear registration aka spatial normalisation. FMRIB Technical Report TR07JA2, 28 June 2007. Available at: http://www.fmrib.ox.ac.uk/datasets/techrep/ tr07ja2/tr07ja2.pdf

17. Burgel U, Amunts K, Hoemke L, et al. White matter fiber tracts of the human brain: Three-dimensional mapping at microscopic resolution, topography and intersubject variability. Neuroimage 2006; 29(4): 1092-1105.

18. Hua K, Zhang J, Wakana S, et al. Tract probability maps in stereotaxic spaces: Analyses of white matter anatomy and tract-specific quantification. Neuroimage 2008; 39(1): 336-347.

19. Benjamini $Y$ and Hochberg Y. Controlling the false discovery rate: A practical and powerful approach to multiple testing. J R Stat Soc Ser B 1995; 57(1): 289-300.

20. Kuhle J, Disanto G, Dobson R, et al. Conversion from clinically isolated syndrome to multiple sclerosis: A large multicentre study. Mult Scler 2015; 21(8): 1013-1024.

21. Brownlee WJ, Miszkiel KA, Altmann DR, et al. Periventricular lesions and MS diagnostic criteria in young adults with typical clinically isolated syndromes. Mult Scler 2017; 23: 1031-1034.

22. Tintore M, Rovira A, Rio J, et al. Defining high, medium and low impact prognostic factors for developing multiple sclerosis. Brain 2015; 138(Pt 7): 1863-1874.

23. Ranjeva JP, Pelletier J, Ibarrola D, et al. MRI/MRS of corpus callosum in patients with clinically isolated syndrome suggestive of multiple sclerosis. Mult Scler 2003; 9: 554-565.

24. Bester M, Heesen C, Schippling S, et al. Early anisotropy changes in the corpus callosum of patients with optic neuritis. Neuroradiology 2008; 50(7): 549-557.

25. Jafari N, Kreft KL, Flach HZ, et al. Callosal lesion predicts future attacks after clinically isolated syndrome. Neurology 2009; 73(22): 1837-1841.

26. Sinnecker T, Oberwahrenbrock T, Metz I, et al. Optic radiation damage in multiple sclerosis is associated with visual dysfunction and retinal thinning-An ultrahigh-field MR pilot study. Eur Radiol 2015; 25(1): 122-131. 
27. Lobsien D, Ettrich B, Sotiriou K, et al. Whole-brain diffusion tensor imaging in correlation to visualevoked potentials in multiple sclerosis: A tract-based spatial statistics analysis. AJNR Am J Neuroradiol 2014; 35(11): 2076-2081.

28. Pagani E, Filippi M, Rocca MA, et al. A method for obtaining tract-specific diffusion tensor MRI measurements in the presence of disease: Application to patients with clinically isolated syndromes suggestive of multiple sclerosis. Neuroimage 2005; 26(1): 258-265.

29. Schmierer K, Wheeler-Kingshott CAM, Boulby PA, et al. Diffusion tensor imaging of post mortem multiple sclerosis brain. Neuroimage 2007; 35(2): 467-477.

30. Filippi M, Rocca MA, Barkhof F, et al. Association between pathological and MRI findings in multiple sclerosis. Lancet Neurol 2012; 11(4): 349-360.
31. Evangelou N, Konz D, Esiri MM, et al. Regional axonal loss in the corpus callosum correlates with cerebral white matter lesion volume and distribution in multiple sclerosis. Brain 2000; 123(9): 1845-1849.

32. Ciccarelli O, Werring DJ, Barker GJ, et al. A study of the mechanisms of normal-appearing white matter damage in multiple sclerosis using diffusion tensor imaging: Evidence of Wallerian degeneration. $J$ Neurol 2003; 250(3): 287-292.

33. Gabilondo I, Martínez-Lapiscina EH, Martínez-Heras E, et al. Trans-synaptic axonal degeneration in the visual pathway in multiple sclerosis. Ann Neurol 2014; 75(1): 98-107.
Visit SAGE journals online journals.sagepub.com/ home/msj

@ SAGE journals 